\title{
Thank you to our reviewers
}

The $B D J$ is always looking for new reviewers to assess manuscripts in all areas of dentistry.

If you would like to be added to our reviewer database, please send an email outlining your areas of expertise to:

Laura Pacey

Editorial Assistant

I.pacey@nature.com

The $B D$ J editorial team would like to thank all the people who help maintain the quality of scientific papers in the $B D J$ by acting as reviewers. Over 1,000 manuscripts were submitted to the $B D J$ this year. The following list contains the reviewers who have worked on scientific papers during 2011.

Liam Addy

Aws Alani

Sondos Albadri

Edith Allen

Frances Andreasen

George Aristidou

Jeremy Bagg

Paul Baker

Graham Ball

Mary Colette Balmer

David Bartlett

Markus Bastir

Paul Batchelor

Kalpesh Bavisha

Habib Benzian

Malcolm Bishop

Anthony Blinkhorn

Stephen Bonsor

Veronica Booth

Elizabeth Bower

Edward Boyle

Liam Boyle

Henk Brand

David Brennan

Alison Bullock

Trevor Burke

Michael Burrow

Robert Chate

Ivor Chestnutt

William Cissell

Paul Coulthard

StJohn Crean

David Croser

Chris Cunningham

Blanaid Daly

Stephen Davies

Scott Deacon

Chris Deery

Hugh Devlin

Thomas Dietrich

Mervyn Druian

Stephen Dunne
Justin Durham

Thomas Dyer

Janice Ellis

Lester Ellman

Jane Evans

Patricia Evans

Josiah Eyeson

Clovis Faggion

Maurice Faigenblum

David Felix

Chris Franklin

Ruth Freeman

Jennifer Gallagher

Matthew Garnett

Paul Gill

Nicholas Goodger

Mark Greenwood

Akil Gulamali

Jennifer Harris

Peter Heasman

Ellie Heidari

Martin Hobdell

Alexander Holden

Christopher Holmgren

Colin Hopper

Peter Howard-Williams

Francis Hughes

Andrew Hyatt

Nicola Innes

Robert Jagger

Waseem Jerjes

Colwyn Jones

Judith Jones

Richard Kahan

Anup Karki

Elizabeth Kay

George Konstantinou

Stephen Lambert-Humble

David Landes

Jason Leitch

Michael Lennon

Ronnie Levine
Debbie Lewis

Michael Lewis

Howard Lloyd

Matthew Locke

Friederike Luther

Christopher Lynch

lain Mackie

Michaelina Macluskey

Isobel Madden

Nicholas Malden

Philip Marsh

Michael Martin

Nicolas Martin

Roger Matthews

W. D. McCall Jr

Bernard McCartan

Robert McCormick

Shane McCrea

Mark McGurk

Niall McLeod

Christopher Mercer

Peter Milgrom

Declan Millett

John Milne

Rebecca Moazzez

Colin Murray

Philip Newsome

Tim Newton

Hien Ngo

Michael Norton

Christopher Orr

Nikolaus Palmer

Caroline Pankhurst

Benjamin Peretz

Nicolaos Pigadas

Stephen Porter

Philip Preshaw

lain Pretty

Stephen Prime

Sobia Rafique

Andrew Rawlinson

John Renshaw
Tara Renton

Scott Rice

Wayne Richards

Peter Robinson

Stephen Robinson

Stuart Robson

Mel Rosenberg

Margaret Ross

Vivian Rushton

Jonathan Sandy

W. P. Saunders

Kevin Seymour

Shakeel Shahdad

David Shaw

Abdullah Shehu

Andrew Smith

Paul Speight

Cathy Stillman-Lowe

John Sweet

Murray Thomson

Martin Tickle

Christopher Tredwin

Anthony Walmsley

Saman Warnakulasuriya

Robert Wassell

Keith Webster

Nicola West

Eric Whaites

David Williams

Mike Williams

Derrick Willmot

Kathy Wilson

David Wray

Callum Youngson

Yehuda Zadik

Thank you also to those who have written a commentary for a research summary and to those who reviewed a manuscript towards the end of this year whose name may not appear above. 